\title{
Lengua
}

\section{Eslovaquismos y bohemismos en español}

\author{
Bohdan ULAŠIN \\ Universidad Comenio de Bratislava \\ bohdan.ulasin@gmail.com
}

Recibido: Octubre de 2010

Aceptado: Enero de 2011

\section{Resumen}

El artículo recoge y analiza desde un punto de vista etimológico, semántico y formal las palabras de origen eslovaco y checo en el vocabulario de la lengua española.

Palabras clave: eslovaquismo, bohemismo, préstamo, calco, epónimo.

Slovakisms and Bohemisms in Spanish

\begin{abstract}
The article collects and analyzes from the etymological, semantic and formal point of view the words of Slovak and Czech origin in the Spanish language.
\end{abstract}

Key words: slovakism, bohemism, loanword, calque, eponym.

En eslovaco y en checo hay centenares de palabras que se originaron en español o que entraron en las lenguas europeas a través del castellano. En el primer grupo registramos palabras como pasodoble, korida, siesta, gitara / kytara ${ }^{1}$, flamenco y en el segundo los indigenismos de las lenguas amerindias como los siguientes: tabak / tabák, kanibal, čokoláda, kakao, kojot, kondor, lama, pampa, koka, pončo, maté, etc. Pero, ¿qué ocurre en la situación inversa?

Los bohemismos y los eslovaquismos se encuentran en el grupo de los eslavismos, préstamos de las lenguas eslavas al vocabulario castellano. Hay que decir que su presencia en español no es en absoluto numerosa, la mayoría de los eslavismos proviene sin lugar a dudas del ruso (vodka, zar, gulag, perestroika, etc.). No obstante, existen en castellano también palabras del eslovaco y del checo, y su recopilación y consiguiente análisis formal, semántico y etimológico será el objetivo de este trabajo.

\footnotetext{
${ }^{1}$ Si hay diferencias entre el eslovaco y el checo, la forma eslovaca aparece en primer lugar y tras una barra la sigue la forma checa. También cabe explicar que bajo la palabra originada en castellano entendemos la unidad de forma y significado. Por lo tanto se mencionan palabras como gitara cuya forma viene en última instancia del griego kithárā, no obstante el significado moderno surgió en español (REJZEK 2001: 328).
} 
En primer lugar nos gustaría definir qué es lo que entendemos bajo el término de eslovaquismo o bohemismo. Las palabras que aparecen en nuestro artículo pertenecen a una de estas seis categorías: checa:

1. Eslovaquismos / bohemismos etimológicos: palabras de etimología eslovaca y

robot 'aparato electromecánico programable' (del checo robot, derivación regresiva de robota 'faena' < paleoeslavo * orbiti)

Aquí se incluyen también las palabras de forma eslovaca y checa cuyo significado ha surgido en otras lenguas:

dobro 'guitarra resofónica', es un acrónimo surgido del nombre de la empresa del inventor John Dopyera (nació como Ján Dopjera en Dolná Krupá, Eslovaquia) que la empezó a fabricar con sus hermanos en la empresa DOpyera BROthers (Los Hermanos Dopyera) ${ }^{2}$

pistola 'arma de fuego corta', (francés pistole < checo piśt'ala 'arma de fuego', el significado originario es 'flauta', 'caña')

2. Eslovaquismos / bohemismos exógenos: palabras que a pesar de su etimología no eslovaca / checa fueron creadas en una de estas lenguas eslavas:

ferritina 'proteina globular que almacena el hierro en los organismos de los vertebrados', fue descubierta por el académico checo Vilém Laufberger en el año 1934 (ferritín < latín ferrum 'hierro')

3. Mezcla de los dos primeros tipos: una palabra derivada o compuesta con un elemento eslavo y uno extranjero:

slavikita f'mineral de la clase de los sulfatos, $\mathrm{MgFe}_{3}\left[(\mathrm{OH})_{3}\left(\mathrm{SO}_{4}\right)_{4}\right] \cdot 18 \mathrm{H}_{2} \mathrm{O}$ ' (slavik- $<$ del nombre propio František Slavík + -ita sufijo latino con el cual se derivan las denominaciones de los minerales)

4. Calcos estructurales léxicos de las palabras o expresiones creadas en las lenguas eslavas (sea de etimología eslovaca / checa o no):

revolución de terciopelo 'cambio no violento del régimen checoslovaco en el año 1989 (calco de Zamatová revolúcia / Sametová revoluce)

5. Calcos semánticos de las palabras creadas en las lenguas eslavas (sean de etimología eslovaca / checa o no):

tunelar en su acepción de 'defraudar los fondos comunes' (del eslovaco y checo tunelovat' / tunelovat < inglés tunnel 'perforar túneles')

6. Eslovaquismos / bohemismos "geográficos"; palabras de etimología y creación no eslovaca / checa cuyo único lazo con el checo o el eslovaco es que su forma proviene de un topónimo del territorio de las Repúblicas Checa y Eslovaca actuales; aun teniendo en cuenta que son las palabras con el menor índice de "eslovaquidad"

\footnotetext{
2 Normalmente se considera un anglicismo:

http://www.etymonline.com/index.php?search=dobro\&searchmode=none, [13-12-2010]
} 
o "bohemidad" y que hay más razones para identificarlas con adquisiciones de otras lenguas, aparecen en nuestro trabajo:

bohemio 'persona de vida irregular y poco estable, especialmente cuando se trata de artistas o escritores' (francés bohème < Bohême 'Bohemia' < lat. Bo(io)hemia: Boio- 'país de la tribu celta de los boyos' + -hemia < germánico *haima 'casa', 'patria')

dólar 'nombre de la moneda oficial de varios países', (del alemán Thaler, su nombre proviene de una mina en Joachimsthal, en checo Jáchymov, en la parte occidental de Bohemia)

En lo que se refiere a la frecuencia, entre los más conocidos y usados encontramos sólo los bohemismos, concretamente las palabras robot, dólar, pistola y sus derivaciones. Las demás palabras forman parte de las terminologías de diferentes disciplinas científicas, se usan en contextos muy especializados y por lo tanto periféricos, p.ej.:

mineralogía: koktaita, vrbaita, hodrushita, slavikita, libethenita...

historia: husita, taborita, revolución de terciopelo...

música, baile: dobro, polca, fujara...

cinología: tchuvatch...

medicina: acatisia...

gastronomía: Becherovka, Budweiser, Pilsen, liptauer, slivovitz..

En la vigésima segunda edición del diccionario de la $\mathrm{RAE}^{3}$ figuran tan sólo las siguientes palabras de nuestra lista: bohemio, dólar, husita, obús, pistola, polca, redova, robot, tálero.

La mayoría de los bohemismos y eslovaquismos que hemos encontrado son sustantivos (robot, dobro, tchuvatch, trdelnik, polca, pistola...) o sintagmas nominales (revolución de terciopelo) y el verbo tunelar. Algunos luego sirven de base para la formación de otras palabras: robot $\rightarrow$ robotizar, robotización, robótica, autorrobot...

Es interesante la observación de que gran parte de estas palabras sean epónimos, o sea apelativizaciones de nombres propios, sea de personas (husita, slavikita, koktaita, bukovskyita, Becherovka...), regiones, países o ciudades (bohemio, Pilsen, dólar, liptauer, hodrushita, kutnahorita, taborita, semtex...).

\section{Checo}

absurdistán $m$ 'régimen, sociedad, país absurdo' (checo absurdni 'absurdo' $<$ lat. absurdus + -stán sufijo persa que significa 'país', 'estado', lo encontramos en los nombres de varios países asiáticos: Kazajstán, Pakistán, Afganistán, etc. ${ }^{4}$ )

Palabra que pasó al inglés a través de las obras de Václav Havel $(* 1936)$, escritor, expresidente de Checoslovaquia (1989-1992) y de la República Checa (19932003). Con esta palabra Havel se refería a su país bajo el régimen comunista alu-

\footnotetext{
${ }^{3} \mathrm{http} / / /$ www.rae.es/rae.html [17-12-2010]

${ }^{4}$ Los datos de la explicación etimológica vienen de Rejzek (2001) y Lutterer - Kropáček - Huňáček (1976). 
diendo a un sinfín de situaciones absurdas y sin sentido a las que él y sus conciudadanos tenían que enfrentarse cada día.

acatisia $f$ 'inhabilidad de permanecer en un sitio o de pie sin moverse, miedo a sentarse' (griego a- 'negación' + griego káthisis 'sesión')

Término acuñado en el año 1901 por Ladislav Haškovec (1866-1944), médico y profesor de neuropatología en la Universidad Carolina de Praga ${ }^{5}$.

becherovka $m$ 'licor de una selección secreta de 32 hierbas, contiene un 38\% de alcohol' (del nombre propio Josef Becher)

Nombrado según su creador, el farmacéutico Josef Becher de Karlovy Vary (ciudad al noroeste de Bohemia) en 1807. Su único productor es la empresa Karlovarská becherovka, SA.

bohemio 'persona de vida irregular y poco estable, especialmente cuando se trata de artistas o escritores' (francés bohème < Bohême 'Bohemia' < lat. Bo(io)hemia: Boio'país de la tribu celta de los boyos' + -hemia < germánico *haima 'casa', 'patria')

En francés se denominó primero con esta palabra a los gitanos de los que se creía que venían de esta parte de Europa. Luego pasó a referirse a una persona (sobre todo a un artista) de vida desordenada, también con el matiz de sufrir penurias económicas.

Budweiser $m$ 'cerveza producida en la ciudad de České Budějovice' (del topónimo Budweis)

El nombre de la cerveza es originariamente un gentilicio alemán budweiser que viene de la denominación alemana de la ciudad de České Budějovice (Böhmisch) Budweis. En checo la cerveza se llama Budvar que es un cruce de dos palabras: Budějovický pivovar 'Fábrica de Cerveza de Budějovice'.

bukovskyita $f$ 'mineral, $\mathrm{Fe}_{2}\left(\mathrm{AsO}_{4}\right)\left(\mathrm{SO}_{4}\right)(\mathrm{OH}) .7 \mathrm{H}_{2} \mathrm{O}$ ' (del nombre propio Bukovský)

Esta denominación proviene del nombre propio del químico checo Antonín Bukovský (1865-1950).

cyrilovita f 'mineral, $\mathrm{NaFe}^{3+}{ }_{3}\left(\mathrm{PO}_{4}\right)_{2}(\mathrm{OH})_{4} \cdot 2\left(\mathrm{H}_{2} \mathrm{O}\right)$ ' (del topónimo Cyrilov)

El nombre viene de la localidad checa de Cyrilov.

dólar $m$ 'nombre de la moneda oficial de varios países'6 (del topónimo Joachimsthal)

\footnotetext{
5 http://www.neuro.lf1.cuni.cz/docs/hask_vecer.pdf [13-12-2010]

${ }^{6}$ Australia, Bahamas, Barbados, Belice, Bermudas, Brunéi, Canadá, Caribe del Este, Ecuador, El Salvador, Estados Unidos, Fiyi, Guyana, Hong Kong, Islas Caimán, Islas Cook, Islas Salomón, Jamaica, Liberia, Malasia, Namibia, Nueva Zelanda, Panamá, Puerto Rico, Samoa, Singapur, Surinam, Trinidad y Tobago, Zimbabue http://es.wikipedia.org/wiki/Dólar [15-12-2010].
} 
Su nombre viene de una mina en Joachimsthal en la parte occidental de Bohemia (en checo se llama Jáchymov). En el siglo XVI estaba bajo la jurisdicción de los condes de Schlick, en su condado se empezó a acuñar una moneda con la plata extraída de sus minas. Este tipo de moneda, que llevaba la efigie de San Joaquín, se denominó Joachimsthaler, nombre que fue pronto abreviado a Thaler, de este vocablo proceden el tálero, que circuló en Alemania hasta 1873, el dólar y el tólar esloveno (GARCÍA-CASTAÑÓN 2001: 50).

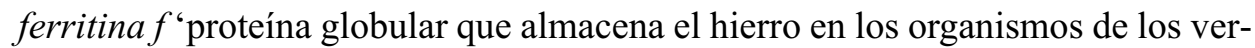
tebrados' (checo ferritín < latín ferrum 'hierro')

Fue descubierta por el académico checo Vilém Laufberger en el año 1934.

furiant $m$ 'baile popular checo, de ritmo $2 / 4 \mathrm{y}^{3 / 4}$ ' (lat. furians $<$ furiare 'ponerse furioso')

Es un baile de gran viveza rítmica, de ahí su nombre. Los ejemplos más conocidos se hallan en las obras de los compositores checos Antonín Dvořák y Bedřich Smetana.

háček $m$ 'signo diacrítico para indicar la palatalización consonántica' (checo háček con la acepción originaria de 'gancho pequeño' < hák 'gancho' < alto alemán medio hāke(n), cf. alemán Haken, inglés hook)

Se utiliza en el alfabeto latinoeslavo (checo, eslovaco, alto lusaciano, croata, esloveno) en los siguientes caracteres ( $\breve{c}, \breve{s}, \breve{z}, \check{r}, t^{\prime}, l^{\prime}$, d', $\check{n}$, ě), pero también la encontramos en lituano, finlandés, estonio, carelio y en algunas lenguas sami, hasta en lakota. Evolucionó de la forma de un punto que se escribía sobre las letras para indicar su palatalización. Fue introducido en la ortografía checa por Jan Hus en su obra De Ortographia Bohemica (1412). Hasta hoy día se conserva en el polaco el tipo más antiguo en forma de un punto: $\dot{z}$. Son sinónimos los términos anticircunflejo o carón.

heyrovskita f'mineral, $\mathrm{Pb}_{10} \mathrm{AgBi}_{5} \mathrm{~S}_{18}$ ' (del nombre propio Jaroslav Heyrovský)

Su denominación deriva del nombre del químico checo Jaroslav Heyrovský (1890-1967).

husita m; adj. 'con esta palabra se refiere al movimiento reformador eclesiástico y al mismo tiempo social y político en la Bohemia del siglo XV' (del nombre propio Jan Hus)

El nombre proviene del teólogo bohemio Jan Hus (1370-1415) que fue condenado a muerte en el Concilio de Constanza.

kankita f'mineral, $\mathrm{Fe}^{3+}\left(\mathrm{AsO}_{4}\right) \cdot 3.5\left(\mathrm{H}_{2} \mathrm{O}\right)$ ' (del topónimo Kaňk $)$

El nombre viene de la localidad checa de Kaňk.

koktaita f'mineral cuya fórmula química es $\left(\mathrm{NH}_{4}\right)_{2} \mathrm{Ca}\left(\mathrm{SO}_{4}\right)_{2} \cdot\left(\mathrm{H}_{2} \mathrm{O}\right)$ ' (del nombre propio Jaroslav Kokta)

Este mineral fue nombrado según el químico checo Jaroslav Kokta (1904-1970). 
kolache / kolace / kolachky m 'tipo de pastel típico de la República Checa' (checo koláče, la forma del plural de koláč 'pastel', koláčky es la forma diminutiva, todo derivado de kolo 'círculo' por su forma < paleoeslavo *kolo)

Se trata de un pequeño panecillo o bollo redondo hecho de masa de levadura, con fruta en almíbar o mermelada en el centro o como relleno. Hora)

kutnahorita $f$ 'mineral, $\mathrm{CaMn}^{2+}{ }_{0.6} \mathrm{Mg}_{0.3} \mathrm{Fe}^{2+}{ }_{0.1}\left(\mathrm{CO}_{3}\right)_{2}$ ' (del topónimo Kutná

Nombrado según la ciudad checa de Kutná Hora.

obús $m$ 'tipo de pieza de artillería' (checo houfnice $<$ houf 'muchedumbre' < alto alemán medio hüfe)

Originariamente era un tipo de catapulta de piedras de los ejércitos husitas del siglo XV. Pilsen)

Pilsener, Pilsen 'tipo de cerveza pale lager de fermentación baja' (del topónimo

Empezó ahemia, en alemán Pilsen).

pistola f 'arma de fuego corta, se usa con una mano' (francés pistole $<$ checo pišstala 'arma de fuego'; el significado originario es 'flautilla', 'caña').

Era un tipo de arma de fuego de los husitas?.

polca f'danza popular de Bohemia', 'música para esta danza' (de půlka 'mitad' o de Polák 'polaco')

La polca apareció en la primera mitad del siglo XIX, algunos piensan que su nombre deriva de la palabra půlka 'mitad' por su ritmo de $2 / 4$, otros dicen que viene del nombre de los polacos, en memoria de su aplastada insurrección contra la dictadura zarista en 1830, hecho que tuvo mucha repercusión entre los checos. En tal caso el motivo sería expresar la solidaridad con el pueblo polaco.

redova f'danza popular checa', 'música de esta danza' (checo rejdová < adjetivo derivado de rejd, probablemente del alto alemán medio reide 'vuelta')

Esta danza checa tiene un compás de 3/4. Se extendió en América Latina (sobre todo en México y en la región andina).

remoska f 'hornillo eléctrico' (acrónimo de las palabras revize, montáže, opravy, servis 'revisión, montajes, reparaciones, servicio' + el sufijo sustantivador $-k a$ )

Es un hornillo eléctrico portátil, inventado a principios de los cincuenta por Oldřich Homuta ${ }^{8}$.

\footnotetext{
7 Existen teorías alternativas de su origen, p.ej. según el nombre de la ciudad italiana de Pistoia donde se fabricaban armas de fuego: http://www.etymonline.com/index.php?term=pistol [15-12-2010].

$8 \mathrm{http}: / / w w w . i n o v a c e . c z / f o r-l i f e / z d r a v i-a-z i v o t n i-s t y l / c l a n e k / c e s k e-i n o v a c e$-remoska-dobyla-svet/ [17-12-2010]
} 
robot $m$ 'aparato electromecánico programable, capaz de manipular objetos' (del checo robot, derivación regresiva de robota 'faena' < paleoeslavo *orbiti 'trabajar')

La palabra proviene de la obra del autor checo Karel Čapek (1890-1938) R.U.R. (Rossum's Universal Robots) que se estrenó en 1921. En un principio Karel Čapek quiso llamar a los robots "laboři" (del latín labor), pero después fue su hermano Josef Capek quien le sugirió la palabra "roboti" proveniente de la raíz eslava robota.

rosickyita f 'mineral, S' (del nombre propio Vojtěch Rosický)

$\mathrm{Su}$ denominación deriva del nombre del mineralogista checo Vojtěch Rosický (1880-1942).

sekaninaita $f$ 'mineral, $\mathrm{Fe}^{2+}{ }_{1.5} \mathrm{Mg}_{0.5} \mathrm{Al}_{4} \mathrm{Si}_{5} \mathrm{O}_{18}$ ' (del nombre propio Josef Sekanina)

$\mathrm{Su}$ denominación deriva del nombre del mineralogista checo Josef Sekanina (1901-1986).

semtex $m$ 'explosivo plástico' (cruce de palabras: topónimo Semtín + exploze 'explosión' < lat. explōsiō)

Explosivo plástico de uso general. Su nombre deriva de la ciudad checa de Semtín donde empezó a fabricarse en el año 1964, actualmente se fabrica en la ciudad de Brno en la República Checa.

slavikita f 'mineral de la clase de los sulfatos, $\mathrm{MgFe}_{3}\left[(\mathrm{OH})_{3}\left(\mathrm{SO}_{4}\right)_{4}\right] \cdot 18 \mathrm{H}_{2} \mathrm{O}$ ' (del nombre propio František Slavik)

La denominación deriva del nombre del mineralogista checo František Slavík (1876-1957).

slivovitz / slivovice $m$ 'aguardiente destilado y fermentado del zumo de ciruelas' (checo slivovice < slíva 'ciruela'; del checo pasó también al alemán austríaco donde se germanizó su ortografía en Slibowitz, de ahí la forma slivovitz) ${ }^{9}$

Bebida típica de Europa central, oriental y suroriental.

taborita 'miembro de la comunidad sectaria de los husitas' (del topónimo Tábor)

Los miembros de este movimiento radical religioso y social surgieron y se organizaron tras ser quemado en la hoguera Jan Hus (1415), se instalaron a unos 70 kilómetros de Praga y fundaron la ciudad de Tábor cuyo nombre deriva del monte bíblico de Tabor.

tálero $m$ (véase dólar)

tólar m 'nombre de la moneda oficial de Eslovenia' (véase más en dólar)

\footnotetext{
${ }^{9}$ En algunas fuentes se afirma que es una palabra del croata o serbio, sin embargo en tal caso debería pronunciarse [ $\int$ livovitz] porque tanto en serbio como en croata se pronuncia una consonante fricativa postalveolar sorda $\int:$ šljivovica.
} 
tschermigita f 'mineral, $\left(\mathrm{NH}_{4}\right) \mathrm{Al}\left(\mathrm{SO}_{4}\right)_{2} \bullet 12\left(\mathrm{H}_{2} \mathrm{O}\right)$ ' (del topónimo Tschermich)

Nombrado según la forma alemana Tschermich de la localidad checa de Čermíky.

vánočka f 'pastel checo' (checo vánočka derivado de Vánoce 'Navidad' < medio préstamo medio calco del alemán Weihnachten con la traducción bohemizante de -nachten en -noce)

Un pastel trenzado, rico en mantequilla y huevo, típico de Navidad.

vrbaita f 'mineral, $\mathrm{Tl}_{4} \mathrm{Hg}_{3} \mathrm{Sb}_{2} \mathrm{As}_{8} \mathrm{~S}_{2} 0$ ' (del nombre propio Karel Vrba)

Nombrado según el mineralogista checo Karel Vrba $(* 1845$ - †1922).

\section{Eslovaco}

bryndza f 'queso blando y salado de leche de oveja' (bryndza < rumano brânză 'queso')

El vocablo fue llevado al territorio eslovaco por los valacos, pastores carpáticos, que llegaron a los Cárpatos eslovacos entre los siglos XV y XVII (BOTÍK 1995: 285). Es de suponer que la palabra, que primitivamente tenía el significado genérico de 'queso', adquirió su significado específico en el territorio de la Eslovaquia de hoy, ya que empieza a fabricarse en 1787 en Detva, Eslovaquia Central. El fundador de la fábrica se llamaba Ján Vagač y se dice que inventó el proceso de cómo ablandar el queso de oveja ${ }^{10}$. A partir del año 2008 está protegida por la UE, figura en el Registro de Denominaciones de Origen Protegidas de la Comisión Europea bajo el nombre de Slovenská bryndza 'Bryndza Eslovaca'11.

dobro $m$ 'guitarra resofónica' (del nombre de la empresa Dopyera Brothers)

Instrumento musical inventado por John Dopyera en 1928 en California (nació como Ján Dopjera en Dolná Krupá ${ }^{12}$, Eslovaquia) que la empezó a fabricar con sus hermanos en la empresa DOpyera BROthers 'Los Hermanos Dopyera', de ahí el acrónimo. Dobro es a la vez un juego de palabras, porque dobro en eslovaco significa la bondad, es decir, lo bueno, como lo afirma uno de los primeros eslóganes publicitarios de la empresa: "Dobro means good in any language" "Dobro significa bueno en cualquier lengua'.

fujara $f$ 'tipo de flauta pastoril' (fujara quizás de los verbos onomatopéyicos fujat', fujačit' 'soplar', 'hacer viento')

La fujara es un aerófono de madera. Alcanza tres octavas. Está compuesta por dos tubos, la longitud del tubo más grande oscila entre 1,60 y 2 metros y tiene tres agujeros. Se originó y se usa tradicionalmente en las regiones de la Eslovaquia

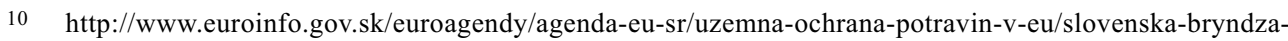
675.html [17-12-2010]

11 http://eur-lex.europa.eu/LexUriServ/LexUriServ.do?uri=OJ:L:2008:189:0019:0020:ES:PDF [17-12-2010].

12 Una aldea a quince kilómetros de la ciudad eslovaca de Trnava donde se celebra cada año el festival internacional de música Dobrofest, www.dobrofest.sk, en honor de John Dopyera y su invento; en esta ciudad también podemos encontrar el museo dedicado a este instrumento.
} 
Central: Banská Bystrica, Podpol'anie y Kokava. En las últimas décadas se va difundiendo por el mundo teniendo ya una posición estable en el estilo musical sincrético llamado world music. En 2005 fue incluida en la lista de Las Obras Maestras del Patrimonio Oral e Intangible de la Humanidad de la UNESCO ${ }^{13}$.

hodrushita f'mineral, $\mathrm{Cu}_{8} \mathrm{Bi}_{12} \mathrm{~S}_{22}$ ' (del topónimo Hodruša)

Nombrado según la ciudad eslovaca de Hodruša.

libethenita f'mineral, $\mathrm{Cu}_{2}\left(\mathrm{PO}_{4}\right)(\mathrm{OH})$ ' (del topónimo Libethen)

Su nombre viene de la forma alemana de la ciudad eslovaca de Lubietová.

liptauer m 'crema de queso' (del topónimo Liptau)

El liptauer está hecho de bryndza (queso de leche de oveja), mantequilla, cebolla muy picada, sal, pimienta, pimentón y mostaza. Está difundido en el territorio del antiguo Imperio Austro-Húngaro, en las cocinas eslovaca, austríaca, húngara. Probablemente la palabra significaba al principio sólo bryndza que se importaba de Liptov a Viena, el nombre es el gentilicio alemán derivado de la forma alemana de la región del septentrión eslovaco Liptau < esl. Liptov. En eslovaco el liptauer se llama bryndzová nátierka, literalmente 'crema de untar de bryndza' o regionalmente šmírkas (cf. en húngaro liptói túro, polaco ser liptowski).

\section{*para) \\ parenica $m$ 'queso tradicional eslovaco' (derivado de para 'vapor' < paleoeslavo}

Un queso semiduro, normalmente ahumado, que se fabrica de leche de oveja. Se produce en cintas de queso que se enrollan en unos rollos parecidos a caracoles. En 2007 recibió la marca de Indicación Geográfica Protegida de la Comisión Europea bajo el nombre de Slovenská parenica ${ }^{14}$.

tchuvatch $m$ 'raza de perro pastor' (čuvač viene de čuvy, palabra obsoleta con el significado de 'nervios', 'sentidos', derivada del verbo čut' 'oír', 'sentir')

Un perro de tamaño grande (de altura de hasta 70 centímetros) formado y criado en las montañas eslovacas, su utilización era la de guardar la casa y defender el rebaño, de ahí también su nombre. La raza fue homologada en 196415 .

trdelnik $m$ 'pastel tradicional' (trdelník derivado de trdlo 'pincho de madera' en el cual está enrollado el pastel)

Un pastel de masa de harina enrollado en un pincho que se asa sobre las brasas. Su forma es cilíndrica y el interior es hueco. En 2007 recibió la marca de Indicación Geográfica Protegida de la Comisión Europea bajo el nombre de Skalický trdelník (Skalica es una ciudad al oeste de Eslovaquia) ${ }^{16}$.

\footnotetext{
$13 \mathrm{http} / / /$ portal.unesco.org/es/ev.php-URL_ID=30973\&URL_DO=DO_TOPIC\&URL_SECTION=201.html [17-12-2010]

$14 \mathrm{http} / / /$ eur-lex.europa.eu/LexUriServ/LexUriServ.do?uri=OJ:C:2007:249:0026:0030:ES:PDF [17-12-2010]

$15 \mathrm{http}: / /$ www.adiestramientodeperros.com/fci142.html [op. cit. 17-12-2010]

16 http://eur-lex.europa.eu/JOHtml.do?uri=OJ\%3AL\%3A2007\%3A330\%3ASOM\%3AES\%3AHTML [17-12-2010]
} 


\section{Checo y eslovaco}

Figuran aquí las palabras cuyo origen no se puede distinguir con seguridad, son conceptos surgidos en la época de Checoslovaquia y a su desarrollo o invención contribuyeron ambas comunidades lingüísticas de la Checoslovaquia unida.

divorcio de terciopelo 'disolución pacífica de Checoslovaquia' (calco de zamato$v y ́$ / sametový rozvod: zamat / samet véase revolución de terciopelo + rozvod: palabra compuesta de roz- 'des-' < antiguo eslavo raz-< paleoeslavo *orz- + -vod de viest' / vést 'conducir', 'llevar' < paleoeslavo *vesti)

Checoslovaquia se separó en dos Repúblicas hereditarias: en la República Checa y la República Eslovaca. El 1 de enero de 1993 se escindió de forma pacífica a diferencia del caso de la ex Yugoslavia.

panelaky $\mathrm{m} \mathrm{pl}$. 'casas de paneles de hormigón prefabricadas' (paneláky, forma del plural de panelák, forma coloquial univerbizada de panelový dom 'casa de paneles', todo de panel < alemán Paneel < alemán bajo medio pan(n)ēl 'revestido de madera', 'panel' < francés antiguo panel 'trozo de paño', 'tableta, plaquita', es la forma diminutiva de pan < lat. pannus 'paño')

En el siglo XX, sobre todo durante la época socialista, en la arquitectura paisajista de Checoslovaquia empieza a predominar un fenómeno urbanístico, los panelaky. Son una especie de bloques de pisos que se construían en las afueras de las ciudades y hasta hoy día forman una parte indisoluble no sólo de las Repúblicas Checa y Eslovaca sino de toda la zona de la antigua Europa socialista. Se suele emplear en plural con la terminación del plural eslava $-y$.

revolución $f$ de terciopelo / revolución tierna ${ }^{17}$ 'caída del régimen socialista checoslovaco en 1989' (calco de zamatová revolúcia / sametová revoluce: adjetivo derivado de zamat / samet 'terciopelo' < nuevo alto alemán Sammet $<$ francés antiguo samit $<$ latín medieval samitum, (e)xamitum $<$ griego medieval hexámiton + latín tardío revolūtiō)

Se refiere al cambio de régimen pacífico y no violento. Cabe decir que en checo predomina el término Sametová revoluce y en eslovaco Nežná revolúcia 'Revolución Tierna'.

tunelar 'defraudar los fondos comunes' (tunelovat / tunelovat' < inglés tunnel 'perforar túneles')

La palabra se originó a principios de los noventa en Checoslovaquia, de ahí pasó a los artículos en lengua inglesa especializados en economía y del inglés llegó al español. Empezó a usarse con más frecuencia durante la crisis económica en Asia a finales de los noventa.

${ }^{17}$ A base de esta expresión se estableció la tradición de darles nombres a las revoluciones cuyo objetivo es derribar al régimen totalitario: Revolución Naranja en Ucrania, Revolución Violeta en Georgia, Revolución Verde en Irán. 


\section{Referencias bibliográficas:}

BOTÍK, J., SLAVKOVSKÝ, P. (dir.) (1995): Encyklopédia l’udovej kultúry Slovenska II, Veda, Bratislava.

GARCÍA-CASTAÑÓN, S. (2001): Diccionario de epónimos del español, TREA, Gijón.

LUTTERER, I., KROPÁČEK, L., HUŇÁČEK, V. (1976): Původ zeměpisných jmen, Mladá Fronta, Praha.

REJZEK, J (2001): Český etymologický slovnik, Leda, Praha.

\section{Referencias en línea:}

Adiestramiento de perros: http://www.adiestramientodeperros.com/fcil42.html [17-12-2010]

Diario Oficial de la Unión Europea:

http://eur-lex.europa.eu/LexUriServ/LexUriServ.do?uri=OJ:L:2008:189:0019:0020:ES:PDF [17-122010]

http://eur-lex.europa.eu/JOHtml.do?uri=OJ\%3AL\%3A2007\%3A330\%3ASOM\%3AES\%3AHTML

[17-12-2010]

http://eur-lex.europa.eu/LexUriServ/LexUriServ.do?uri=OJ:C:2007:249:0026:0030:ES:PDF [17-122010]

Euroinfo:http://www.euroinfo.gov.sk/euroagendy/agenda-eu-sr/uzemna-ochrana-potravin-veu/slovenska-bryndza-675.html [17.12.2010]

Inovace: http:/www.inovace.cz/for-life/zdravi-a-zivotni-styl/clanek/ceske-inovace-remoska-dobyla-svet/ [17-12-2010]

Neurologická klinika Univerzity Karlovy v Praze:

http://www.neuro.lf1.cuni.cz/docs/hask_vecer.pdf [13-12-2010]

Online Etymology Dictionary:

http://www.etymonline.com/index.php?search=dobro\&searchmode=none, [13-12-2010];

http://www.etymonline.com/index.php?term=pistol [15-12-2010].

Real Academia Española: http://www.rae.es/rae.html [17-12-2010]

Unesco:http://portal.unesco.org/es/ev.php

URL_ID=30973\&URL_DO=DO_TOPIC\&URL_SECTION=201.html [17.12.2010]

Wikipedia: http://es.wikipedia.org/wiki/Dólar [15-12-2010]. 